For Internal Distribution Only

Accelerator Division

Alternating Gradient Synchrotron Department BROOKHAVEN NATIONAL LABORATORY

Upton, New York 11973

Accelerator Division

Technical Note

AGS/AD/Tech. Note No. 420

\title{
THE BUCKET AND THE BUNCH WITH A \\ SECOND RF HARMONIC
}
A. Luccio

October 18, 1995 


\section{THE BUCKET AND THE BUNCH WITH A 2.ND RF HARMONIC}

\section{A.Luccio}

In the presence of a second RF harmonic the voltage acting on a particle is

(1)

$$
e V=e V_{1} \sin \left(\omega_{R F} t+\varphi_{1}\right)+e V_{2} \sin \left(2 \omega_{R F} t+\varphi_{2}\right)
$$

With the positions

$$
V_{0} \equiv V_{1}, \quad \chi=\frac{V_{2}}{V_{o}}, \quad \phi=\omega_{R F} t+\varphi_{1}, \quad \Delta \varphi=\varphi_{2}-2 \varphi_{1}
$$

the differential equation for the energy change during the acceleration becomes

$$
\tau \frac{d}{d t}(\Delta E)=e V_{o}\left\{\sin \phi-\sin \phi_{s}+\chi\left[\sin (2 \phi+\Delta \varphi)-\sin \left(2 \phi_{s}+\Delta \varphi\right)\right]\right\}
$$

with $\phi_{s}$ the synchronous phase, to be calculated by solving the transcendental Equation

$$
\frac{\Delta E_{s}}{e V_{0}}=\sin \phi_{s}+\chi \sin \left(2 \phi_{s}+\Delta \varphi\right) \text {. }
$$

To find the bucket separatrix -the "bucket"-, let us start from the differential equation for the phase

$$
\frac{d^{2} \phi}{d t^{2}}=\frac{2 \pi}{\tau^{2}} \frac{h \eta}{\beta^{2}} \frac{e V_{0}}{E_{s}} f_{b}
$$

with $f_{b}$ the function on the rhs of Eq. (3)

$$
f_{b}=\sin \phi-\sin \phi_{s}+\chi\left[\sin (2 \phi+\Delta \varphi)-\sin \left(2 \phi_{s}+\Delta \varphi\right)\right]
$$

and with the harmonic number $h$, the dispersion $\eta$, and the relativistic velocity $\beta$ evaluated for the synchronous particle (we dropped the subscript "s"). 
An integral of motion is found by integration in the standard way ${ }^{1}$

(7)

$$
\int \frac{d^{2} \phi}{d t^{2}} \frac{d \phi}{d t} d t=\frac{2 \pi}{\tau^{2}} \frac{h \eta}{\beta^{2}} \frac{e V_{0}}{E_{s}} \int f_{b} \frac{d \phi}{d t} d t
$$

The integration yields the following

$$
\frac{K}{2}\left(\frac{\Delta E}{E_{s}}\right)^{2}+e V_{0} \tilde{b}\left(\phi, \phi_{s}\right)=H=\text { const },
$$

with

(9)

$$
K=2 \pi \frac{h \eta}{\beta^{2}} E_{s}
$$

and

$$
\tilde{b}\left(\phi, \phi_{s}\right)=\cos \phi+\phi \sin \phi_{s}+\chi\left[\frac{1}{2} \cos (2 \phi+\Delta \varphi)+\phi \sin \left(2 \phi_{s}+\Delta \varphi\right)\right] .
$$

The constant $H$ of Eq.(3) is the Hamiltonian of the system

$$
H=\frac{1}{2} K W^{2}+U
$$

and the bucket is found using the two fixed points $\phi_{s}$ and $\pi-\phi_{\mathrm{s}}$ of the distribution ${ }^{2}$

$$
H(\Delta E, \phi)=H\left(0, \pi-\phi_{s}\right)
$$

The resulting bucket is

$$
\begin{aligned}
& b\left(\phi, \phi_{s}\right)=\cos \phi+\phi \sin \phi_{s}+\cos \phi_{s}-\left(\pi-\phi_{s}\right) \sin \phi_{s} \\
& +\chi\left[\frac{1}{2} \cos (2 \phi+\Delta \varphi)+\phi \sin \left(2 \phi_{s}+\Delta \varphi\right)-\cos \left(2 \phi_{s}-\Delta \varphi\right)-\left(\pi-\phi_{s}\right) \sin \left(2 \phi_{s}+\Delta \varphi\right)\right]
\end{aligned}
$$

1 D.A.Edwards and M.J.Syphers An Introduction to the Physics of High Energy Accelerators Wiley, NY 1993, p.38

2 J.M.Kats Synchronous Particle and Bucket Dynamics AGS/AD/89-1, BNL-52171, October 3, 1988 
To accommodate the bunch in the bucket, we want to find the two extreme points of the separatrix, $\phi_{1}$ and $\phi_{2}\left(\phi_{2}<\phi_{1}\right)$. With some manipulation of Eq. (13), a convenient form of the transcendental Equation to be solved to find these points, using as a parameter . the bunch length $\Delta \phi$ can be written as

$$
\sin \psi=A-\frac{\chi}{4 \sin \left(\frac{1}{2} \Delta \phi\right)} \sin (2 \psi+\Delta \varphi) \text {, }
$$

with the positions

$$
\phi_{1}=\psi+\frac{\Delta \phi}{2}, \quad \phi_{2}=\phi_{1}-\Delta \phi, \quad A=\frac{\frac{1}{2} \Delta \phi}{\sin \left(\frac{1}{2} \Delta \phi\right)}\left[\sin \phi_{s}+\chi \frac{1}{2} \Delta \phi \sin \left(2 \phi_{s}+\Delta \phi\right)\right]
$$

Eq.(4) for the synchronous phase and Eq.(14) for the bucket extreme points can be solved numerically. A very efficient and fast routine for this task is the Newton-Raphson iteration, based on the simple algorithm

$$
x_{n+1}=x_{n}-\frac{f}{f^{\prime}},
$$

that solves the Equation $f \approx 0-f^{\prime}$ is the derivative of $f$ - A convenient starting point for the NR iteration, for Eq. (4) is the synchronous phase with no second harmonic

$$
\phi_{s}^{(1)}=\operatorname{arsin} \frac{\Delta E_{s}}{e V_{0}}
$$

(actually a point slightly beyond $\phi_{s}$ ). A starting point for solving Eq. (14) is

$$
\phi_{1}^{(1)}=\frac{1}{2} \Delta \phi+\operatorname{ar} \sin \left(\frac{\frac{1}{2} \Delta \phi}{\sin \frac{1}{2} \Delta \phi} \sin \phi_{s}\right)
$$

Indeed, if $\chi=0$, the exact solutions of Eqs. (4) and (14) are given by Eqs. (17) and (18), respectively.

Examples of how the synchronous phase is changing by adding a second harmonic to the RF field are shown in Figures 1 and 2. The stable phase is found as the (first positive) zero point of these curves. Figures 3, 4 and 5 show buckets. Parameters are: 


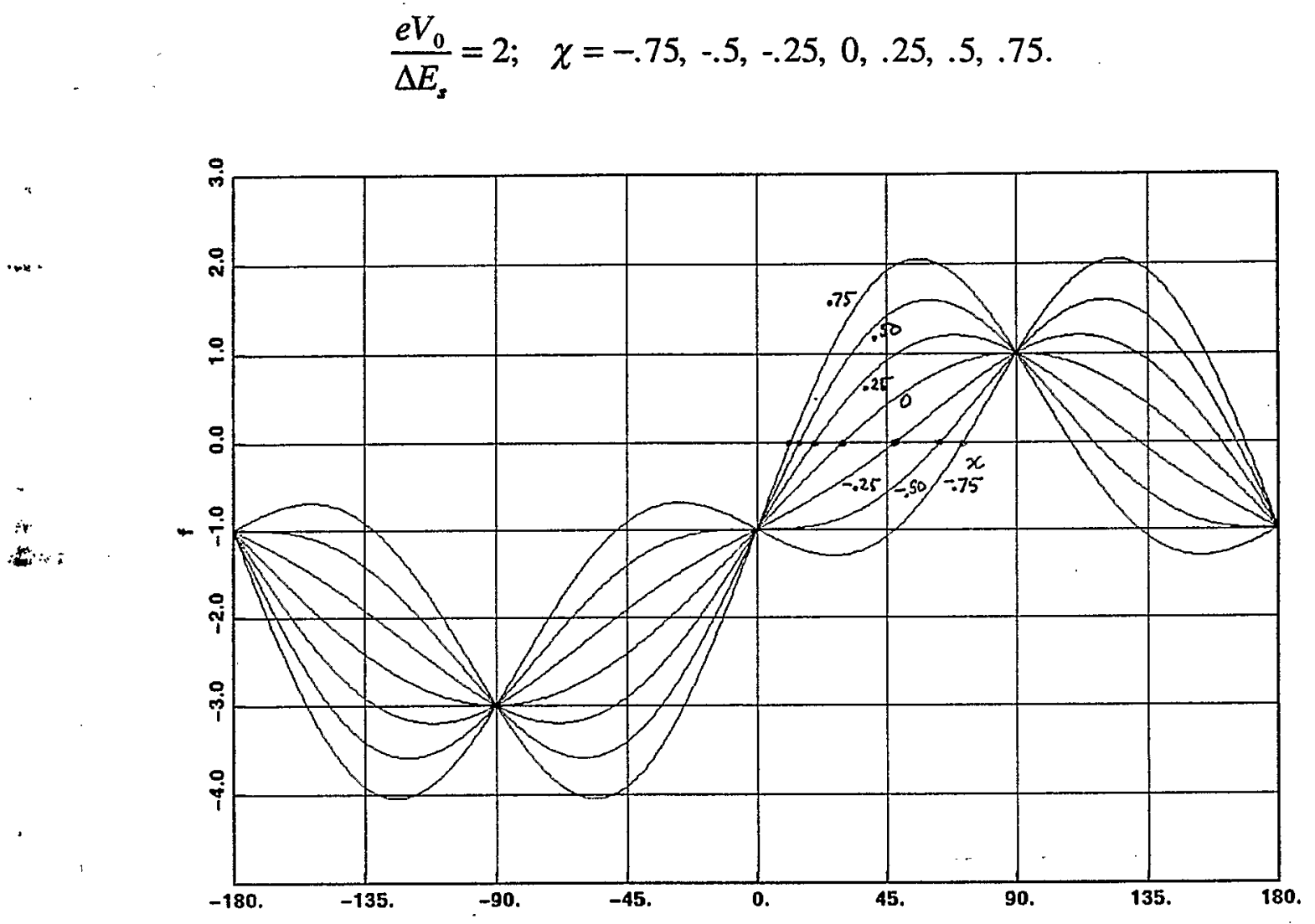

Fig. 1. Synchronous phase $(f=0) . \Delta \phi=0^{\circ}$.

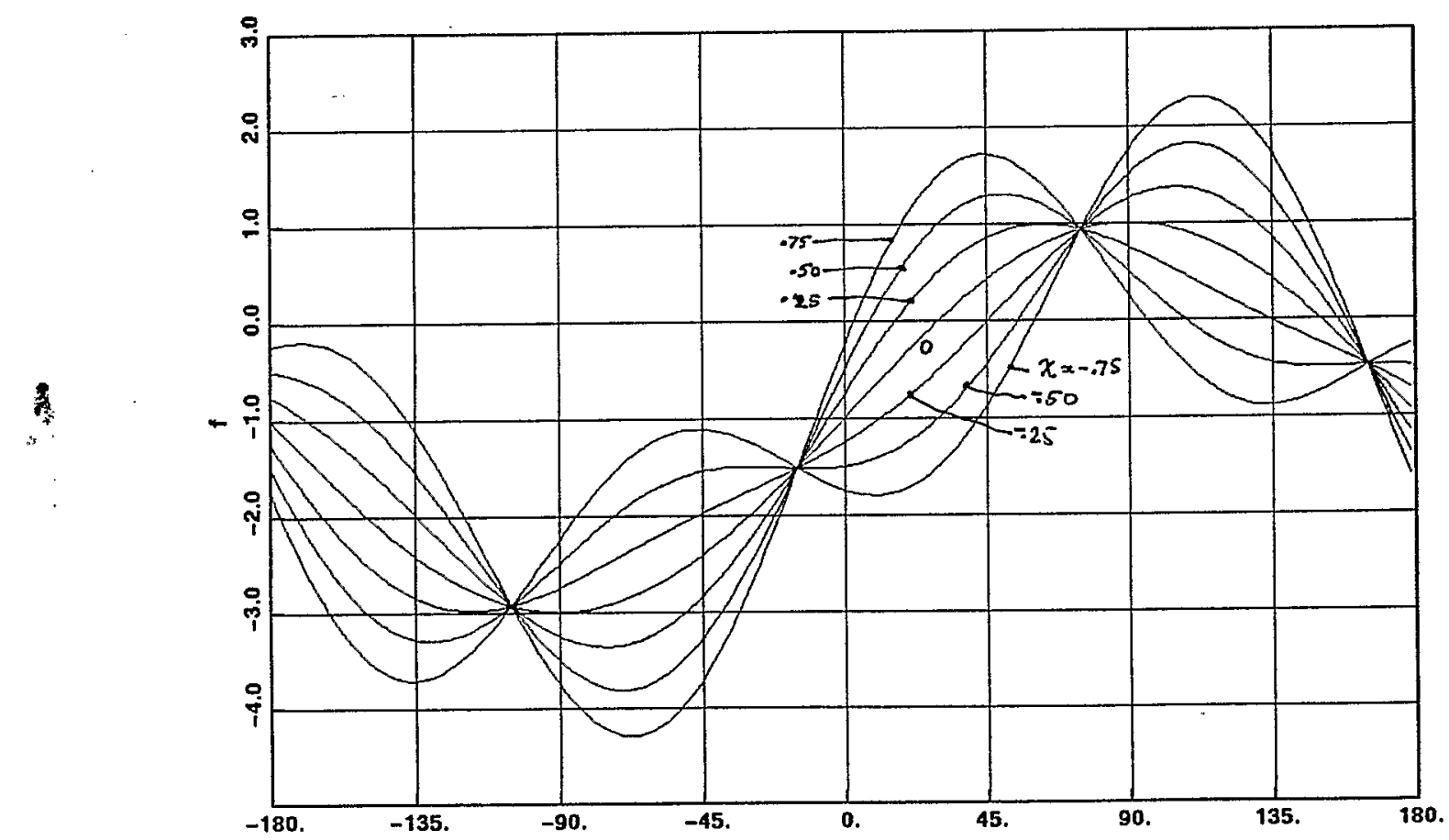

Fig. 2. Synchronous phase $(f=0) . \Delta \phi=30^{\circ}$. 


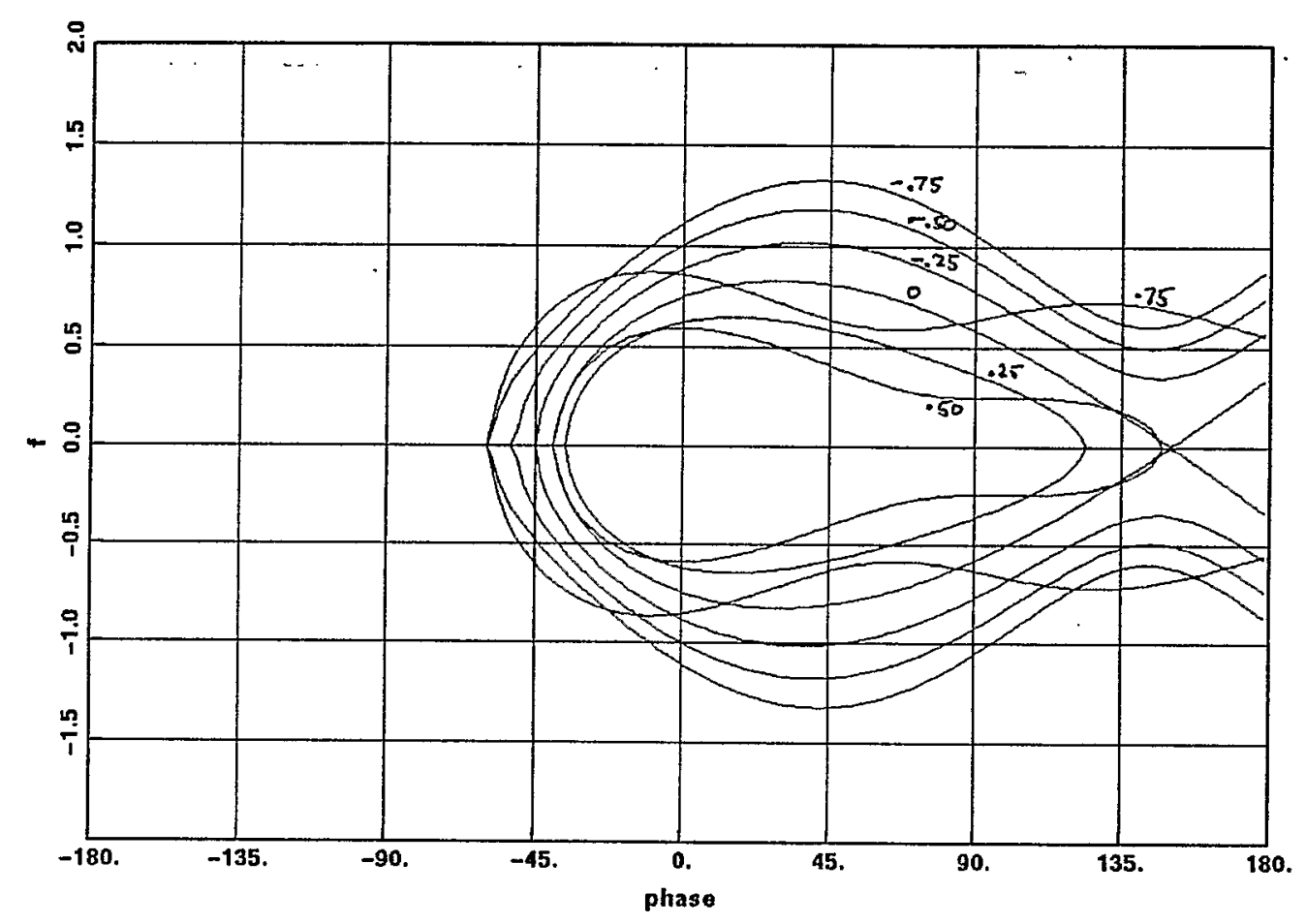

Fig. 3. Buckets: $\Delta \phi=-30^{\circ}$.

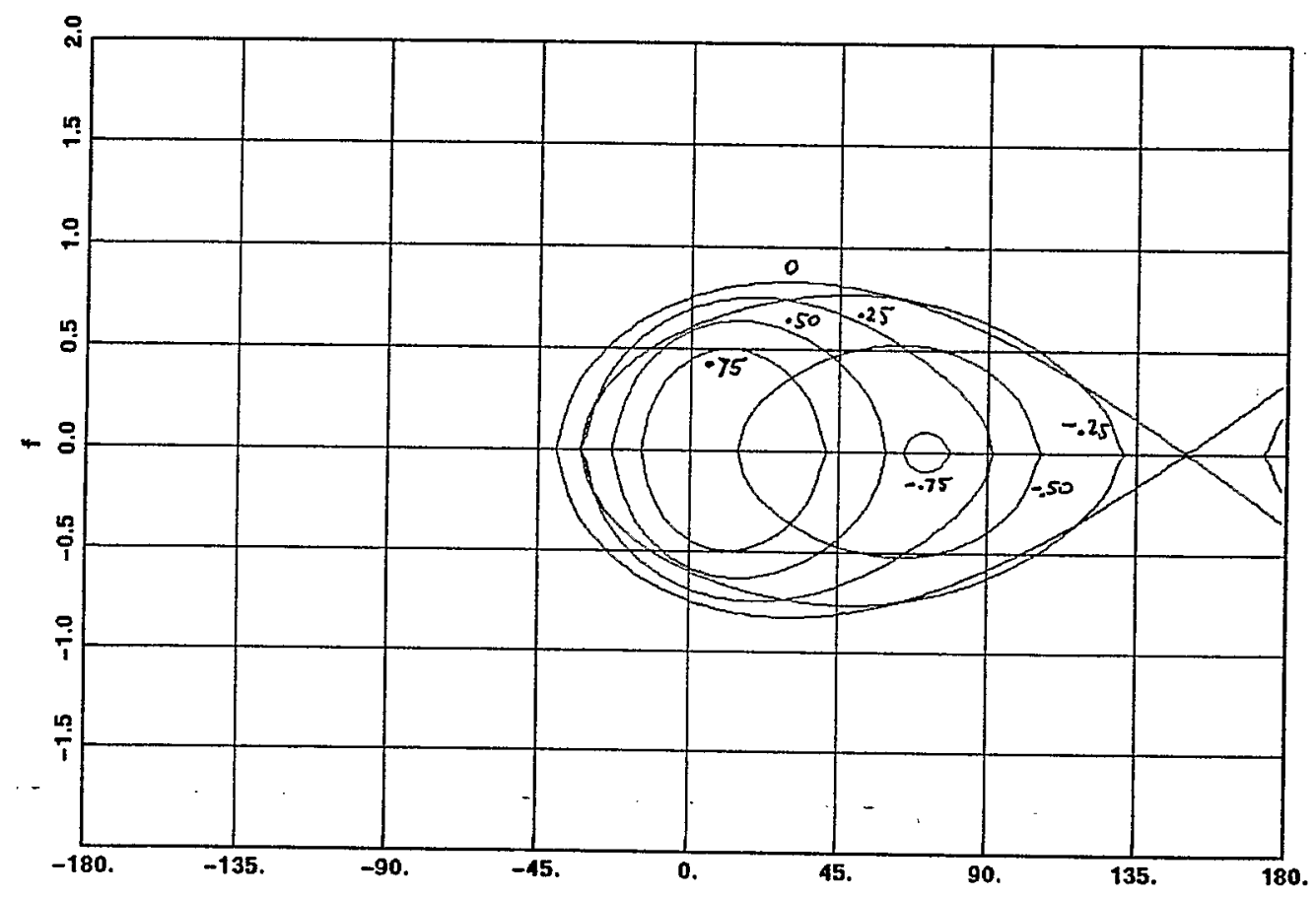

Fig. 4. Buckets: $\Delta \phi=0^{\circ}$. 


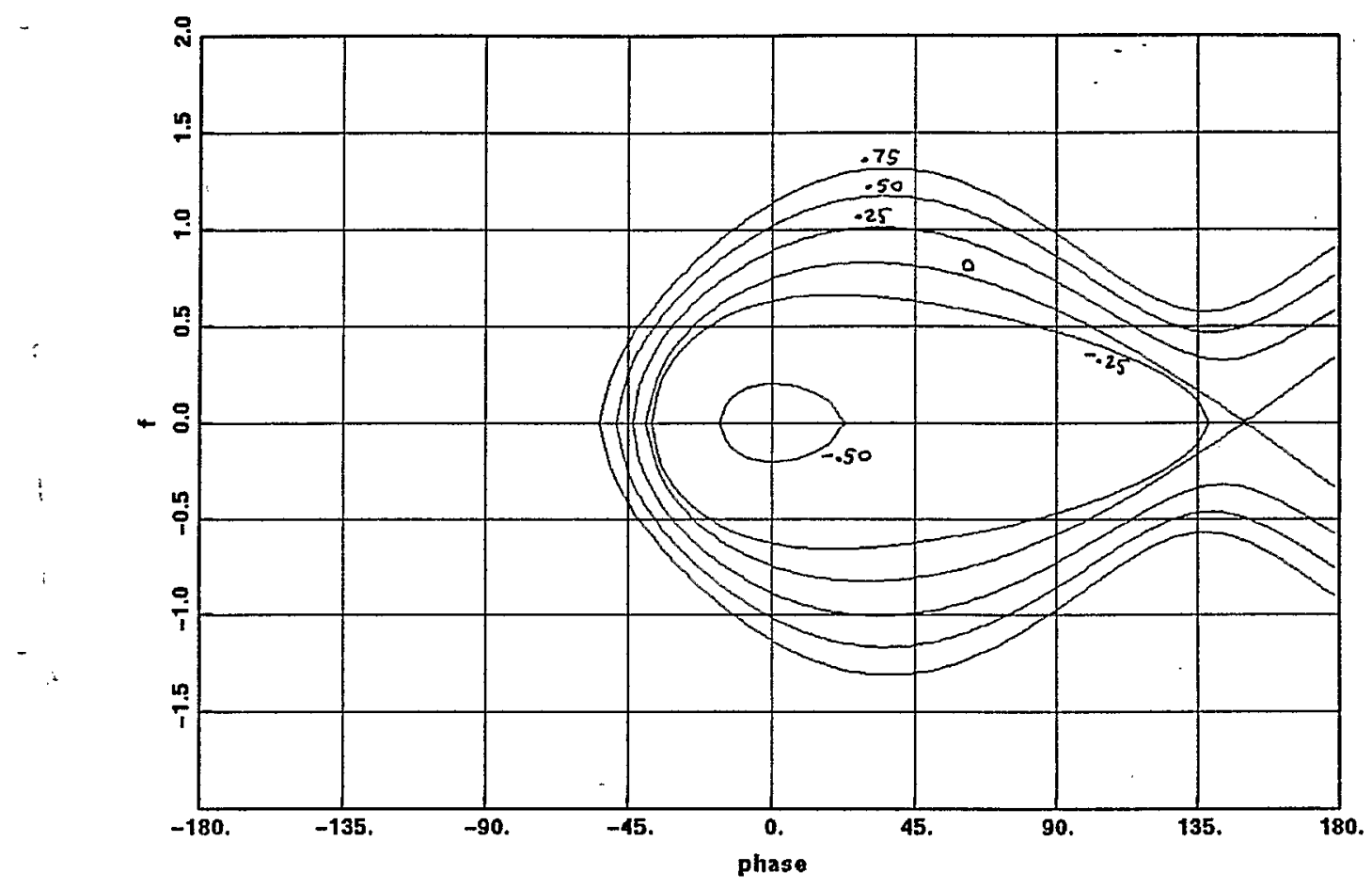

Fig. 5. Buckets: $\Delta \phi=30^{\circ}$. 